\title{
Efectos del entrenamiento vibratorio sobre el riesgo de caída en adultos mayores institucionalizados: una revisión breve
}

\author{
Effects of vibration training on the fall risk in institutionalized older adults: \\ a brief review \\ *Jordan Hernández-Martínez, *Rodrigo Ramírez-Campillo
}

Hernández, J. \& Ramírez, R. (2017). Efectos del entrenamiento vibratorio sobre el riesgo de caída en adultos mayores institucionalizados: una revisión breve. Revista Ciencias de la Actividad Física UCM, $\mathrm{N}^{\circ}$ 18(2) julio-diciembre, 1-7.

\begin{abstract}
RESUMEN
Objetivo: analizar los efectos del entrenamiento vibratorio sobre el riesgo de caída, el cual implica mediciones de velocidad de caminata, equilibrio, potencia y fuerza muscular, en adultos mayores institucionalizados, mediante una revisión sistemática. Método: se realizó una búsqueda bibliográfica sistemática en PUBMED, una de las bases de datos de disponibilidad electrónica más grandes del mundo. Se seleccionaron estudios clínicos experimentales en Inglés realizados en los últimos 5 años. Resultados: 6 estudios fueron detectados. Entre los principales hallazgos, el entrenamiento vibratorio mejoró la velocidad de caminata, el equilibrio, la densidad ósea, la fuerza y potencia muscular, en combinación con otro ejercicio o de manera individual. Conclusión: el entrenamiento vibratorio en adultos mayores institucionalizados, disminuye el riesgo de caídas en este grupo etario.
\end{abstract}

\section{PALABRAS CLAVE}

Velocidad de caminata, equilibrio, potencia y fuerza muscular.

\begin{abstract}
Objective: to analyze the effects of vibration training on fall risk, which implies measurements in walking speed, balance, power and muscle strength, in institutionalized older adults, through a systematic review. Method: a systematic bibliographical search was carried out in PUBMED, one of the largest electronic availability databases in the world. Experimental clinical studies in English were selected and carried out for a period of five years. Results: Six studies were detected. Among the main findings, vibration training improved walking speed, balance, bone density, power and muscle strength, in combination with another type of exercise or individually, as well. Conclusion: vibration training in institutionalized older adults reduces fall risk for this age group.
\end{abstract}

\section{Key words}

Walking speed, balance, power and muscle strength.

\footnotetext{
* Departamento de Ciencias de la Actividad Física. Núcleo de Investigación en Salud, Actividad Física y Deporte. Universidad de Los Lagos. Osorno, Chile.
} 


\section{INTRODUCCIÓN}

El envejecimiento es un proceso caracterizado por la pérdida de capacidades físicas, cognitivas y funcionales (Nelson et al., 2004.; Nelson et al., 2007), implicando mayor riesgo de caída en adultos mayores pudiendo ocasionar lesiones graves como fracturas óseas y en algunos casos incapacidad a largo plazo, lo que se asocia a una baja densidad mineral ósea, disminución de la calidad de vida, morbilidad y mortalidad (Masud \& Morris, 2001). Observándose en personas mayores institucionalizadas un mayor deterioro osteoarticular en comparación a las no institucionalizadas (Walston et al., 2006), las cuales pueden estar más expuestas a caídas en su vida diaria, la mayoría de estas caídas ocurren en una superficie uniforme, seguida por transferencias al subir a una silla o una escalera, estas son causadas por una pérdida inesperada del equilibrio (Roudsari, Ebel, Corso, Molinari, \& Koepsell, 2005). Investigaciones realizadas en el Reino Unido (Scuffham, Chaplin, \& Legood, 2003), Estados Unidos (Englander, Hodson, \& Terregrossa, 1996) y Australia (Moller, 2004), han demostrado que las caídas son una tremenda carga para los servicios sociales y de salud (Fielden, Purdie, Horne, \& Devane, 2001). Siendo importante incorporar nuevas estrategias para prevenir las consecuencias negativas de las caídas. Una de éstas, es promover el ejercicio físico (American Geriatrics Society. British Geriatrics Society et al., 2001) al aumentar la actividad física mediante un programa de ejercicio físico adecuado, se puede mejorar la autonomía de personas mayores y prevenir las caídas (Cameron et al., 2012; Forster, Lambley, \& Young, 2010; Gill et al., 2002; Howe, Rochester, Neil, Skelton, \& Ballinger, 2011; Kagan \& Puppione, 2011). Estos programas se basan solo en ejercicio físico o estrategias multifactoriales (American Geriatrics Society. British Geriatrics Society et al., 2001) Intervenciones mediante ejercicio físico han resultado eficaces para reducir las caídas y mejorar la capacidad funcional entre personas mayores, incluyen entrenamiento de fuerza y resistencia (Buchner, Cress, de Lateur, \& cols., 1997). Mejorando el equilibrio, la velocidad de caminata y la fuerza muscular en las piernas que están estrechamente relacionadas con el riesgo de caída en adultos mayores (Cameron et al., 2012; Oliver, Bell, Gallagher, \& cols., 2007). Si bien el entrenamiento de fuerza y resistencia han demostrado ser eficaces para la disminución de caídas en adultos mayores, el entrenamiento mediante vibraciones de cuerpo completo ha mostrado mejoras en la velocidad de caminata, el equilibrio, fuerza y potencia muscular del miembro inferior (Bautmans, Van Hees, Lemper, \& Mets, 2005; A. Bogaerts, Delecluse, Claessens, \& cols., 2007.; Madou \& Cronin, 2008; Rees, Murphy, \& Watsford, 2008; SitjàRabert et al., 2012), las cuales han mostrado relación con el riesgo de caída en los adultos mayores.

El objetivo del presente estudio es actualizar los conocimientos realizando una revisión bibliográfica que documente los efectos del entrenamiento vibratorio sobre el riesgo de caída en adultos mayores institucionalizados.

\section{METODOLOGÍA}

Se realizó una revisión bibliográfica entre Agosto del 2017 y Octubre del 2017 en la base de datos de acceso electrónico PubMed, una de las más grandes del Mundo (Choudhri, Siddiqui, Khan, \& Cohen, 2015). Utilizando las siguientes palabras claves: vibration training, institutionalized older adults and risk of falls.

Para seleccionar los estudios fue adoptado como criterio de inclusión i) estudios clínicos experimentales que indiquen los efectos del entrenamiento vibratorio sobre el riego de caída en adultos mayores institucionalizados, ii) publicaciones en Ingles, iii) publicaciones en los últimos 5 años (i.e., 2012-2017). De un total 18 estudios encontrados, 12 estudios fueron excluidos por no cumplir los criterios de inclusión. Por tanto, la presente revisión incluye 6 estudios clínicos experimentales que analizaron los efectos del entrenamiento vibratorio sobre el riesgo de caída en adultos mayores institucionalizados, combinado o no con otro ejercicio físico (Tabla 1). 
Tabla 1

Características experimentales de los estudios seleccionados.

\begin{tabular}{|c|c|c|c|}
\hline Autores & Sujetos & $\begin{array}{l}\text { Características del } \\
\text { entrenamiento con } \\
\text { vibraciones }\end{array}$ & Principales resultados \\
\hline Ross, et al., 2012 & $\begin{array}{l}77 \text { participantes, } 38 \\
\text { participantes ( } 80 \pm 8,6 \text { años) } \\
\text { entrenamiento vibratorio, } \\
39 \text { participantes }(82 \pm 8,1 \\
\text { años) Grupo de ejercicio sin } \\
\text { vibraciones. }\end{array}$ & $\begin{array}{l}\text { Un total de } 8 \text { semanas, } \\
3 \text { sesiones } / \text { semana } / 5 \times 1 \\
\text { min } \times 30 \text { segundos de } \\
\text { descanso/frecuencia } \\
15-30 \mathrm{~Hz} / \text { amplitud } 2 \text { a } \\
8 \mathrm{~mm} .\end{array}$ & $\begin{array}{l}\uparrow \text { Velocidad de caminata } \\
\uparrow \text { Equilibrio } \\
\uparrow \text { Timed and Up Go }\end{array}$ \\
\hline Zhang, et al., 2014 & $\begin{array}{l}44 \text { personas mayores frágiles } \\
(85,27 \pm 3,63 \text { años) un grupo } \\
\text { entrenamiento vibratorio y un } \\
\text { grupo control (ejercicio). }\end{array}$ & $\begin{array}{l}\text { Un total de } 8 \text { semanas, } \\
3 \text { a } 5 \text { sesiones/semana/ } 4 \\
\text { a } 5 \text { series } \times 60 \text { segundos/ } \\
\text { frecuencia } 6 \text { a } 26 \mathrm{~Hz} / \\
\text { amplitud } 1 \text { a } 3 \mathrm{~mm} .\end{array}$ & $\begin{array}{l}\uparrow \text { Timed and Up Go } \\
\uparrow \text { Fuerza en la extensión } \\
\text { de rodilla } \\
\uparrow \text { Estabilidad postural } \\
\uparrow E l \text { estado de salud } \\
\text { general }\end{array}$ \\
\hline Smith, et al., 2016 & $\begin{array}{l}60 \text { participantes, } 36 \text { mujeres } \\
\text { y } 24 \text { hombres ( } 82,2 \pm 4,9 \text { años) } \\
\text { entrenamiento vibratorio } \\
(n=13)(82,2 \pm 5,0 \text { años) } \\
\text { ejercicio combinado con } \\
\text { entrenamiento vibratorio } \\
(n=17)(83,4 \pm 5,0 \text { años), } \\
\text { ejercicio sin vibraciones }(n=16) \\
(80,5 \pm 6,2 \text { años) grupo control } \\
(n=14)(81,7 \pm 5,7 \text { años). }\end{array}$ & $\begin{array}{l}\text { Un total de } 12 \\
\text { semanas } / 2 \text { sesiones/ } \\
\text { semana/ } 5 \text { minutos/ } \\
\text { frecuencia } 30 \mathrm{~Hz} / \\
\text { amplitud } 1-3 \mathrm{~mm} \text {. }\end{array}$ & $\uparrow$ Equilibrio \\
\hline Corrie, et al., 2014 & $\begin{array}{l}61 \text { participantes, ( } 37 \text { mujeres y } \\
24 \text { hombres) }(80,2 \pm 6,5 \text { años })\end{array}$ & $\begin{array}{l}\text { Un total de } 12 \\
\text { semanas } / 3 \text { sesiones/ } \\
\text { semana/ } 6 \times 1 \text { minuto/ } \\
\text { frecuencia } 28,4 \mathrm{~Hz} / \\
\text { amplitud } 1 \text { a } 3 \mathrm{~mm} \text {. }\end{array}$ & $\begin{array}{l}\uparrow \text { Potencia muscular del } \\
\text { miembro inferior } \\
\uparrow \text { Densidad ósea }\end{array}$ \\
\hline Ochi, et al., 2014 & $\begin{array}{l}20 \text { participantes, } 10 \\
\text { participantes }(80,9 \pm 2,8 \text { años) } \\
\text { grupo de entrenamiento } \\
\text { vibratorio, } 10 \text { participantes } \\
(80,2 \pm 3,3 \text { años) grupo de } \\
\text { ejercicio sin vibraciones. }\end{array}$ & $\begin{array}{l}\text { Un total de } 12 \\
\text { semanas/3 sesiones/ } \\
\text { semana/ } 30 \mathrm{~min} / \\
\text { frecuencia } 10-21 \mathrm{~Hz} / \\
\text { amplitud } 3 \text { a } 7 \mathrm{~mm} \text {. }\end{array}$ & $\begin{array}{l}\uparrow \text { Velocidad de caminata } \\
\uparrow \text { Timed and Up Go } \\
\uparrow \text { Fuerza en la extensión } \\
\text { de rodilla } \\
\uparrow \text { Equilibrio }\end{array}$ \\
\hline Sitja, et al., 2014 & $\begin{array}{l}159 \text { participantes, } 107 \text { mujeres } \\
\text { y } 52 \text { hombres, edad media } \\
82 \text { años, } 81 \text { participantes } \\
\text { realizaron Entrenamiento } \\
\text { vibratorio, } 78 \text { participantes } \\
\text { grupo ejercicio sin vibraciones. }\end{array}$ & $\begin{array}{l}\text { Un total de } 6 \text { semanas, } \\
3 \text { sesiones/semana/30 } \\
\text { minutos/frecuencia } \\
30-35 \mathrm{~Hz} / \text { amplitud } 2 \text { a } \\
4 \mathrm{~mm} .\end{array}$ & $\uparrow$ Prueba de Tinetti \\
\hline
\end{tabular}

\section{RESULTADOS}

En el estudio de Ross, Martin, and Newham (2012) se observaron mejoras en el Test Timed and Up Go, velocidad de caminata, equilibrio y una mayor longitud de zancada, post intervención de 8 semanas de entrenamiento vibratorio con adición al ejercicio de fuerza y equi- librio. Zhang et al. (2014) encontraron mejoras luego de una intervención de 8 semanas mediante vibraciones en el Test Timed and Up Go, la fuerza máxima de extensión de rodilla, la estabilidad de la postura y el estado de salud general. Sitjà et al. (2014) encontraron mejoras 
después de una intervención de entrenamiento vibratorio de 6 semanas en la prueba de Tinetti y las 5 pruebas de Sit-to-stand. Smith et al. (2016) observaron mejoras en el equilibrio estático y el autocuidado luego de 12 semanas de entrenamiento vibratorio combinado con otro ejercicio en adultos mayores. Ochi et al. (2014) reportaron mejoras luego de la fase de intervención de 12 semanas mediante entrenamiento vibratorio combinado con otro ejercicio en la velocidad de caminata, Timed and Up Go, fuerza máxima de extensión de rodilla, velocidad de paso y un aumento en la actividad máxima EMG del musculo gastronemio. Mientras que CORRIE et al. (2014) encontraron mejoras luego de una intervención de 12 semanas mediante entrenamiento vibratorio en la potencia muscular del miembro inferior y densidad mineral ósea.

\section{DISCUSIÓN}

El entrenamiento vibratorio disminuiría las caídas en adultos mayores, en combinación con otro ejercicio o de manera individual, siendo una forma de intervención en adultos mayores institucionalizados poco invasiva, la que puede contribuir a una mayor adherencia en este grupo etario. Resultados positivos podrían ser observados desde 6 a 12 semanas de intervención, incluyendo aumentos en la velocidad de caminata, el equilibrio, la fuerza y la potencia muscular. La frecuencia $(\mathrm{Hz})$ y amplitud ( $\mathrm{mm})$ de entrenamiento varió entre cada estudio, pudiendo esto influir en sus resultados.

Se han reportado mejoras en la fuerza y potencia muscular en hombres y mujeres mayores de comunidades institucionalizadas después de una intervención de entrenamiento vibratorio de cuerpo completo (Bautmans et al., 2005; Bogaerts, G., \& cols., 2009). La potencia de las extremidades inferiores está relacionado con el rendimiento funcional en las personas mayores (Bassey et al., 1992), esta mejoría en la potencia muscular podría beneficiar a las actividades en las que la potencia de las piernas puede estar limitando la subida/descenso de escaleras. Los mecanismos por los que el entrenamiento vibratorio de cuerpo completo promueve la fuerza y potencia muscular no están del todo claros, pero aumenta la actividad Electromiográfica de las extremidades inferiores durante los reflejos de estiramiento inducido (Ritzmann, Kramer, Gruber, Gollhofer, \& Taube, 2010). El aumento en la actividad máxima EMG del músculo Gastronemio en la mejora de la flexión plantar podría explicar el aumento en la velocidad de paso y la recuperación de la caída simulada hacia delante proporcionando un impulso durante la recuperación de la caída hacia delante (Thelen et al., 2000). Debido a que la flexión plantar desempeña un papel importante en la movilidad y la estabilidad en personas mayores (Kirkwood, 2011; Suzuki, Bean, \& Fielding, 2001). Cabe destacar que en uno de estos estudios se observó un aumento en la densidad ósea post intervención de entrenamiento vibratorio de cuerpo completo estos hallazgos son muy favorables en la mejora de la calidad de vida en los adultos mayores, debido a que una baja densidad ósea contribuye a una mayor probabilidad de fracturas y una reducción en la calidad de vida (Masud \& Morris, 2001).

\section{CONCLUSIÓN}

A pesar de que los estudios difirieron en el tiempo de las intervenciones, en la frecuencia y amplitud, el entrenamiento vibratorio de cuerpo completo mostró mejoras en la velocidad de caminata, equilibrio y densidad ósea, las cuales están directamente relacionadas con el riesgo de caída en adultos mayores. Por lo tanto, el entrenamiento con vibraciones puede ser una buena opción para mejorar la salud, funcionalidad y disminuir el riesgo de caídas en adultos mayores institucionalizados. 


\section{REFERENCIAS BIBLIOGRÁFICAS}

American Geriatrics Society. British Geriatrics Society, American, Academy, of, Orthopaedic, Surgeons, ... Prevention. (2001). Guideline for the Prevention of Falls in Older Persons. Journal of the American Geriatrics Society, 49(5), 664-672. Retrieved from http://dx.doi. org/10.1046/j.1532-5415.2001.49115.x doi:10.1046/j.1532-5415.2001.49115.x

Bassey, E. J., Fiatarone, M. A., O'neill, E. F., Kelly, M., Evans, W. J., \& Lipsitz, L. A. (1992). Leg extensor power and functional performance in very old men and women. Clinical Science, 82(3), 321-327. doi:10.1042/cs0820321

Bautmans, I., Van Hees, E., Lemper, J.-C., \& Mets, T. (2005). The feasibility of whole body vibration in institutionalised elderly persons and its influence on muscle performance, balance and mobility: a randomised controlled trial [ISRCTN62535013]. BMC Geriatrics, 5, 17-17. doi:10.1186/1471-2318-5-17

Bogaerts, G., C., \& cols. (2009). Effects of whole body vibration training on cardiorespiratory fitness and muscle strength in older individuals (a 1-year randomised controlled trial). Age and Ageing, 38(4), 448-454. doi:10.1093/ageing/afp067

Bogaerts, A., Delecluse, C., Claessens, A., \& cols. (2007.). Impact of whole-body vibration training versus fitness training on muscle strength and muscle mass in Older men: A 1-year randomized controlled trial. J Gerontol A Biol Sci Med Sci, 62, 630-635.

Buchner, D., Cress, M., de Lateur, B., \& cols. (1997). The effect of strength and endurance training on gait, balance, fall risk, and health services use in communityliving older adults. J Gerontol A Biol Sci Med Sci, 52 (4), 218-224.
Cameron, I. D., Gillespie, L. D., Robertson, M. C., Murray, G. R., Hill, K. D., Cumming, R. G., \& Kerse, N. (2012). Interventions for preventing falls in older people in care facilities and hospitals. Cochrane Database of Systematic Reviews(12). doi:10.1002/14651858.CD005465.pub3

Corrie, H., Brooke-Wavell, K., Mansfield, N.J., Cowley, A., Morris,R., Masud, T. (2014). Effects of vertical and sidealternating vibration training on fall risk factors and bone turnover in older people at risk of falls. Age and Ageing, 1-8.

Choudhri, A., Siddiqui, A., Khan, N., \& Cohen, H. (2015). Understanding bibliometric parameters and analysis. Radiographics : a review publication of the Radiological Society of North America. Inc., 35(3). 736-746.

Englander, F., Hodson, T., \& Terregrossa, R. (1996). Economic dimensions of slip and fall injuries. J Forensic Sci, 41, 733746.

Fielden, J., Purdie, G., Horne, G., \& Devane, P. (2001). Hip fracture incidence in New Zealand, revisited. N Z Med J., 13; 114., 154-156.

Forster, A., Lambley, R., \& Young, J. B. (2010). Is physical rehabilitation for older people in long-term care effective? Findings from a systematic review. Age and Ageing, 39(2), 169-175. doi:10.1093/ ageing/afp247

Gill , T. M., Baker , D. I., Gottschalk , M., Peduzzi , P. N., Allore , H., \& Byers, A. (2002). A Program to Prevent Functional Decline in Physically Frail, Elderly Persons Who Live at Home. New England Journal of Medicine, 347(14), 1068-1074. doi:10.1056/NEJ$\underline{\text { Moa020423 }}$ 
Howe, T. E., Rochester, L., Neil, F., Skelton, D. A., \& Ballinger, C. (2011). Exercise for improving balance in older people. Cochrane Database of Systematic Reviews(11). doi:10.1002/14651858. CD004963.pub3

Kagan, S.H., \& Puppione, A.A. (2011). Not preventing falls--promoting function. Geriatric Nursing, 32(1), 55-57. doi:10.1016/j.gerinurse.2010.12.005

Kirkwood, R.N., Trede, R.G., Moreira Bde, S., Kirkwood, S.A., Pereira, L.S. (2011). Decreased gastrocnemius temporal muscle activation during gait in elderly women with history of recurrent falls. Gait \& Posture, 34, 60-64.

Madou, K., \& Cronin, J. (2008). The effects of whole body vibration on physical and physiological capability in special populations. Hong Kong Physiotherapy Journal, 26, 25-38.

Masud, T., \& Morris, R. (2001). Epidemiology of falls. Age and Ageing, 30 (suppl. 4) 3-7.

Moller, J. (2004). Projected costs of fall related injury to older persons due to demographic change in Australia. Commonwealth Department of Health and Ageing.

Nelson, M., Layne, J., Bernstein, M., Nuernberger, A., Castaneda, C., Kaliton, D., . . . Fiataron, S., MA. (2004.). The effects of multidimensional home-bassed exercise on functional performance in elderly people. J Gerontol A Biol Sci Med Sci., 59 (2). 154-160.

Nelson, M., Rejeski, W., Blair, S., Duncan, P., Judge, J., King, A., \& cols. (2007). Physical activity and public health in older adults: recommendation from the American College of Sports Medicine and the American Heart Association. Med Sci Sports Exerc., 39 (8), 14351445.
Ochi, A., Tomokazu Abe, Kazumasa Yamada, Satoko Ibuki, Hiroshige Tateuchi, \& Ichihashi., N. (2014). Effect of balance exercise in combination with wholebody vibration on muscle activity of the stepping limb during a forward fall in older women: A randomized controlled pilot study. Archives of Gerontology and Geriatrics, 60, 244-251.

Oliver, D., Bell, J., Gallagher, D., \& cols. (2007). Development of a pathway to facilitate gastrostomy insertion for patients with MND. Int J Palliat Nurs, 13, 426-429.

Rees, S., Murphy, A., \& Watsford, M. (2008). Effects of whole-body vibration exercise on lower-extremity muscle strength and power in an older population: A randomized clinical trial. Phys Ther., $88,462-470$.

Ritzmann, R., Kramer, A., Gruber, M., Gollhofer, A., \& Taube, W. (2010). EMG activity during whole body vibration: motion artifacts or stretch reflexes? European Journal of Applied Physiology, 110(1), 143-151. doi:10.1007/s00421$\underline{010-1483-\mathrm{x}}$

Ross, D.P., Martin, F.C., \& Newham, D.J. (2012). Whole-body vibration in addition to strength and balance exercise for falls-related functional mobility of frail older adults: a singleblind randomized controlled trial. Clinical Rehabilitation, 26(10), 915923. doi:10.1177/0269215511435688

Roudsari, B.S., Ebel, B.E., Corso, P.S., Molinari, N.-A.M., \& Koepsell, T.D. (2005). The acute medical care costs of fall-related injuries among the U.S. older adults. Injury, 36(11), 1316-1322. doi:10.1016/j. injury.2005.05.024

Scuffham, P., Chaplin, S., \& Legood, R. (2003). Incidence and costs of unintentional falls in older people in the United Kingdom. Journal of Epidemiology and Community Health, 57(9), 740-744. doi:10.1136/jech.57.9.740 
Sitjà-Rabert, M., Rigau, D., Fort Vanmeerghaeghe, A., Romero-Rodríguez, D., Bonastre Subirana, M., \& Bonfill, X. (2012). Efficacy of whole body vibration exercise in older people: a systematic review. Disability and Rehabilitation, 34(11), 883-893. doi:10.3109/0963828 $\underline{8.2011 .626486}$

Sitjà, R., Mercè, Martínez-Zapata, M. J., Fort Vanmeerhaeghe, A., Rey Abella, F., Romero-Rodríguez, D., \& Bonfill, X. (2014). Effects of a Whole Body Vibration (WBV) Exercise Intervention for Institutionalized Older People: A Randomized, Multicentre, Parallel, Clinical Trial. Journal of the American Medical Directors Association, 16(2), 125-131. doi:10.1016/j.jamda.2014.07.018

Smith, D.T., Judge, S., Malone, A., Moynes, R.C., Conviser, J., \& Skinner, J.S. (2016). Effects of bioDensity Training and Power Plate Whole-Body Vibration on Strength, Balance, and Functional Independence in Older Adults. Journal of Aging and Physical Activity, 24(1), 139148. doi:10.1123/japa.2015-0057

Suzuki, T., Bean, J.F., \& Fielding, R.A. (2001). Muscle Power of the Ankle Flexors Predicts Functional Performance in Community-Dwelling Older Women. Journal of the American Geriatrics Society, 49(9), 1161-1167. doi:10.1046/j.15325415.2001.49232.x

Thelen, D., G., Muriuki, M., James, J., Schultz, A. B., Ashton-Miller, J. A., \& Alexander, N. B. (2000). Muscle activities used by young and old adults when stepping to regain balance during a forward fall. Journal of Electromyography \& Kinesio$\log y, 10,93-101$.
Walston, J., Hadley, E.C., Ferrucci, L., Guralnik, J.M., Newman, A.B., Studenski, S.A., . . . Fried, L.P. (2006). Research Agenda for Frailty in Older Adults: Toward a Better Understanding of Physiology and Etiology: Summary from the American Geriatrics Society/National Institute on Aging Research Conference on Frailty in Older Adults. Journal of the American Geriatrics Society, 54(6), 991-1001. doi:10.1111/j.1532-5415.2006.00745.x

Zhang, L., Weng, C., Liu, M., Wang, Q., Liu, L., \& He, Y. (2014). Effect of wholebody vibration exercise on mobility, balance ability and general health status in frail elderly patients: a pilot randomized controlled trial. Clinical Rehabilitation, 28(1), 59-68. doi:10.1177/0269215513492162

\section{Dirección para correspondencia}

Rodrigo Ramírez Campillo Phd.

Department of Physical Activity Sciences

University of Los Lagos (Osorno, Chile)

Campus of Chuyaca

Av. Fuchslocher $n^{\circ} 1305$ (Osorno, Chile)

Postal Code 5290000

Contacto:

r.ramirez@ulagos.cl

https://www.researchgate.net/profile/Rodrigo_Ramirez-Campillo

http://ulagos.cl/

Recibido: 16-10-2017

Aceptado: 20-11-2017 